Pacific Journal of Mathematics

ON RIGHT ZERO UNIONS OF COMMUTATIVE SEMIGROUPS 


\title{
ON RIGHT ZERO UNIONS OF COMMUTATIVE SEMIGROUPS
}

Robert P. Dickinson, JR.

\begin{abstract}
Let $F=\left\{S_{r}: r \in R\right\}$ be a disjoint family of semigroups. One says that $F$ has a right zero union (RZU) if there exists a semigroup $S$ which is a disjoint union of the $S_{r}$ where each $S_{r}$ is a left ideal of $S$. This paper gives some theorems on RZU of commutative semigroups with special emphasis placed on commutative cancellative semigroups.
\end{abstract}

Suppose $S$ is an RZU of commutative cancellative semigroups. It is proven that $S$ has a quotient right abelian group; thus $S$ is left commutative and left cancellative. Conversely, it is proven that if a semigroup $S$ is left commutative and left cancellative, then $S$ is an RZU of commutative cancellative semigroups. Suppose $F$ is a family of commutative semigroups having an RZU; it is proven that a certain family of cancellative homomorphic images of $F$ also has an RZU. Finally, necessary and sufficient conditions are given for a family of commutative cancellative semigroups to have an RZU.

The study of RZU is a special case of the study of "bands of semigroups." R. Yoshida has studied the dual problem of left zero unions.

II. Some necessary conditions for RZU and an embedding result. A semigroup $S$ is left commutative if $x y z=y x z$ for all $x, y$, and $z$ in $S$.

LEMma 2.1. The RZU of two commutative semigroups is left commutative.

Proof. The symmetric conditions $A B \subseteq B, B A \subseteq A, A$ and $B$ are commutative, are given. Let $a \in A$, and let $b, b_{1} \in B$. Now $a b b_{1}=$ $a\left(b b_{1}\right)=a\left(b_{1} b\right)=\left(a b_{1}\right) b=b\left(a b_{1}\right)=b a b_{1}$. Other cases are proven similarly.

DEFINITION 2.2. Let $C$ be a commutative cancellative semigroup. The quotient group, $G$, of $C$ is the smallest group into which $C$ may be injected. If $C \cong T$, a group, then $G \cong\left\{s t^{-1}: s, t \in C\right\}$. Note $G$ is abelian. (For more on quotient groups see [1].)

A right abelian group is the direct product of a right zero semigroup and an abelian group. A quotient right abelian group will have the same meaning as quotient group; namely, the smallest right abelian group into which a semigroup $S$ can be injected.

The next lemma is proven using the following result of Petrich 
[2]: A semigroup $S$ is a semilattice of semigroups each of which is the Cartesian product of rectangular band and a group iff $S$ is a union of groups and its idempotents form a semigroup.

LEMMA 2.3. Let $F=\left\{G_{\alpha}: \alpha \in A\right\}$ be a disjoint family of groups. Then $F$ has an $R Z U$ iff all the $G_{\alpha}$ are isomorphic. If the $R Z U$ exists then it is isomorphic to the right group $G \times A$, where $G_{\alpha} \cong G$, and where $A$ is considered as a right zero semigroup.

Proof. Let $S$ be an RZU of $F$. Certainly $S$ is union of groups. The idempotents of $S$ are exactly the $e_{\alpha}$, where $e_{\alpha}$ is the identity of $G_{\alpha}$. Since $e_{\alpha}$ is an identity and since $G_{\alpha} G_{\beta} \subseteq G_{\beta}$, we have $\left(e_{\alpha} e_{\beta}\right)\left(e_{\alpha} e_{\beta}\right)=$ $e_{\alpha}\left(e_{\beta}\left(e_{\alpha} e_{\beta}\right)\right)=e_{\alpha}\left(e_{\alpha} e_{\beta}\right)=\left(e_{\alpha} e_{\alpha}\right) e_{\beta}=e_{\alpha} e_{\beta}=e_{\beta}$, for $e_{\alpha} e_{\beta}$ is the idempotent of $G_{\beta}$. Thus the idempotents of $S$ form a right zero semigroup. This semigroup is isomorphic to $A$, but also, by Petrich, to a semilattice union $\mathrm{U}_{\gamma \in \Gamma} L_{\gamma} \times R_{\gamma}$, and this implies that $|\Gamma|=1,\left|L_{\gamma}\right|=1$, and $R_{r}=$ A.

THEOREM 2.4. Let $S$ be an $R Z U$ of $F=\left\{C_{\alpha}: \alpha \in A\right\}$, where $F$ is $a$ disjoint family of commutative cancellative semigroups. Let $G_{\alpha}$ be the quotient group of $C_{\alpha}$. We consider the $G$ to be disjoint. Then all the $G_{\alpha}$ are isomorphic, and they have an $R Z U, T$.

$T$ is isomorphic to $G \times A$, where $G_{\alpha} \cong G$, and where $A$ is considered as a right zero semigroup.

Furthermore, $T$ is the quotient right abelian group of $S$ in the following sense. There exists an injection (isomorphism into) $h$ from $S$ into T. If $H \times R$ is any right abelian group into which $S$ can be injected (by $f$, say), then there exists an injection $k: T \rightarrow H \times R$ such that the following diagram commutes:

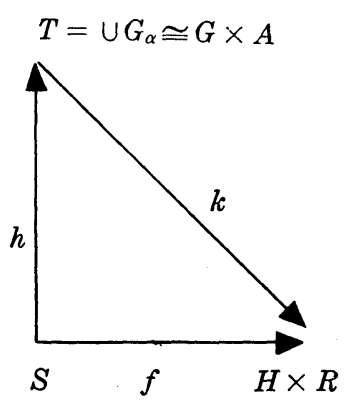

Figure 1

Proof. Let $F^{\prime \prime}=\left\{G_{\alpha}: \alpha \in A\right\}$, where $G_{\alpha}$ is the quotient group of $C_{\alpha}$, and where $G_{\alpha} \cap G_{\beta}=\phi$ if $\alpha \neq \beta$. Each $C_{\alpha}$ may be injected as a 
set of generators into $G_{\alpha}$. Let $h_{\alpha}$ be such an injection: $G_{\alpha}=\left\{h_{\alpha}(s) h_{\alpha}(t)^{-1}\right.$ : $\left.s, t \in C_{\alpha}\right\}$.

Let $T=\bigcup_{\alpha \in A} G_{\alpha}$. We define a semigroup operation $*$ on $T$. With this operation $T$ will be an RZU of $F^{\prime \prime}$. Let $g=h_{\alpha}(s) h_{\alpha}(t)^{-1}$, and let $l=h_{\beta}(u) h_{\beta}(v)^{-1}$.

Let $g * l=h_{\beta}(s \circ u) h_{\beta}(t \circ v)^{-1}$, where $\circ$ is the semigroup operation on S.

Since $s, t \in C_{\alpha}$ and $u, v \in C_{\beta}$, $(s \circ u)$ and $(t \circ v)$ are in $C_{\beta}$. Thus these quantities are in the domain of $h_{\beta}$. We now verify $*$ is well defined.

Suppose $g=h_{\alpha}(s) h_{\alpha}(t)^{-1}=h_{\alpha}(a) h_{\alpha}(b)^{-1}, a, b \in C_{\alpha}$, and $l=h_{\beta}(u) h_{\beta}(v)^{-1}=$ $h_{\beta}(c) h_{\beta}(d)^{-1}, c, d \in C_{\beta}$. We would like to prove that: $h_{\beta}(s \circ u) h_{\beta}(t \circ v)^{-1}=$ $h_{\beta}(a \circ c) h_{\beta}(b \circ d)^{-1}$. Equivalently: $h_{\beta}(s \circ u) h_{\beta}(b \circ d)=h_{\beta}(a \circ c) h_{\beta}(t \circ v)$, or $h_{\beta}((s \circ u) \circ(b \circ d))=h_{\beta}((a \circ c) \circ(t \circ v))$. We now verify that $(s \circ u) \circ(b \circ d)=$ $(a \circ c) \circ(t \circ v)$.

We are given $h_{\alpha}(s) h_{\alpha}(t)^{-1}=h_{\alpha}(a) h_{\alpha}(b)^{-1}$. Equivalently: $h_{\alpha}(s) h_{\alpha}(b)=$ $h_{\alpha}(\alpha) h_{\alpha}(t)$, or $h_{\alpha}(s \circ b)=h_{\alpha}(a \circ t)$. Since $h_{\alpha}$ is $1-1: s \circ b=a \circ t$. Similarly $u \circ d=c \circ v$. Multiply left and right hand sides together: $(s \circ b) \circ(u \circ d)=$ $(a \circ t) \circ(c \circ v)$. These products are taken in the subsemigroup $C_{\alpha} \cup C_{\beta}$ of $S$. By Lemma 2.1, $C_{\alpha} \cup C_{\beta}$ is left commutative. Thus $(s \circ b) \circ(u \circ d)=$ $(s \circ u) \circ(b \circ d)$, and $(a \circ t) \circ(c \circ v)=(a \circ c) \circ(t \circ v)$. Thus $(s \circ u) \circ(b \circ d)=(a \circ c) \circ(t \circ v)$.

It is easily proven that $*$ is associative, and that $*$ restricted to any $G_{\alpha}$ is just the given group operation.

Since $T$ is an RZU of groups, it follows from Lemma 2.3 that $T \cong G \times A$.

The $h$ of the diagram is to be an injection of $S=\mathrm{U}_{\alpha \in A} C_{\alpha}$ into $\mathrm{U}_{\alpha \in A} G_{\alpha}$. Recall that if $\alpha \neq \beta$ then $G_{\alpha} \cap G_{\beta}=\phi$ and $C_{\alpha} \cap C_{\beta}=\phi$. Define $h$ by: $h$ restricted to $C_{\alpha}$ is $h_{\alpha}$. Since $h_{\alpha}$ is $1-1 h$ is $1-1$. Let $x \in C_{\alpha}, y \in C_{\beta}$. We now prove that $h(x \circ y)=h(x) * h(y)$, or $h_{\beta}(x \circ y)=$ $h_{\alpha}(x) * h_{\beta}(y)$. Now $h_{\alpha}(x)=h_{\alpha}(x \circ x) h_{\alpha}(x)^{-1}$ and $h_{\beta}(y)=h_{\beta}(y \circ y) h_{\beta}(y)^{-1}$. Thus $h_{\alpha}(x) * h_{\beta}(y)=h_{\beta}((x \circ x) \circ(y \circ y)) h_{\beta}(x \circ y)^{-1}$. By Lemma 2.1, $(x \circ x) \circ(y \circ y)=$ $(x \circ y) \circ(x \circ y)$. Thus

$$
h_{\alpha}(x) * h_{\beta}(y)=h_{\beta}((x \circ y) \circ(x \circ y)) h_{\beta}(x \circ y)^{-1}=h_{\beta}(x \circ y) h_{\beta}(x \circ y) h_{\beta}(x \circ y)^{-1}=h_{\beta}(x \circ y) \text {. }
$$

Let $f$ be an injection of $S$ into another right abelian group $H \times$ $R$. If $f(x)=(g, r)$ define $f(x)^{-1}=\left(g^{-1}, r\right)$. One proves that $f(x \circ y)^{-1}=$ $f(x)^{-1} f(y)^{-1}$.

We now define $k$ of the diagram. Let $x \in G_{\alpha}$. There exists $s, t \in C_{\alpha}$ such that $x=h_{\alpha}(s) h_{\alpha}(t)^{-1}$. Define $k(x)=f(s) f(t)^{-1}$.

We now verify that $k$ is well defined. Suppose $x=h_{\alpha}(s) h_{\alpha}(t)^{-1}=$ $h_{\alpha}(u) h_{\alpha}(v)^{-1}$. Then $h_{\alpha}(s) h_{\alpha}(v)=h_{\alpha}(u) h_{\alpha}(t)$, or $h_{\alpha}(s \circ v)=h_{\alpha}(u \circ t)$. Since $h_{\alpha}$

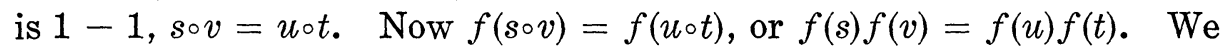
now show that $f(s) f(t)^{-1}=f(u) f(v)^{-1}$.

Let $\pi$ be the projection of $H \times R$ onto $R$, the right zero semi- 
group. Since $C_{\alpha}$ is commutative, $\pi f\left(C_{\alpha}\right)$ is commutative, but then $\left|\pi f\left(C_{\alpha}\right)\right|=1$. Thus $f\left(C_{\alpha}\right) \subseteq H \times\left\{\alpha^{\prime}\right\}=T_{\alpha^{\prime}}$ for some $\alpha^{\prime}$ in $R$.

Since $s, t, u, v$ are in $C_{\alpha}, f(s), f(t), f(u), f(v), f(t)^{-1}$, and $f(v)^{-1}$ are all in $T_{\alpha^{\prime}}$. Since $T_{\alpha^{\prime}}$ is commutative, $f(s) f(v)=f(u) f(t)$ implies $f(s) f(t)^{-1}=f(u) f(v)^{-1}$.

We now verify that the diagram is commutative. Let $s \in C_{\alpha}$. Then $h(s)=h_{\alpha}(s)=h_{\alpha}(s \circ s) h_{\alpha}(s)^{-1} \cdot \quad k(h(s))=f(s \circ s) f(s)^{-1}=f(s) f(s) f(s)^{-1}=$ $f(s)$.

We now verify that $k$ is a homomorphism. Let $x=h_{\alpha}(s) h_{\alpha}(t)^{-1}$, $y=h_{\beta}(u) h_{\beta}(v)^{-1}$. Then $k(x * y)=k\left(h_{\beta}(s \circ u) h_{\beta}(t \circ v)^{-1}\right)=f(s \circ u) f(t \circ v)^{-1}=$ $f(s) f(u) f(t)^{-1} f(v)^{-1}$. Since a right abelian group is left commutative, $k(x * y)=f(s) f(u) f(t)^{-1} f(v)^{-1}=f(s) f(t)^{-1} f(u) f(v)^{-1}=k(x) k(y)$.

We now prove $k$ is $1-1$. We first prove $k$ restricted to $G_{\alpha}$ is $1-1$. Let $x=h_{\alpha}(s) h_{\alpha}(t)^{-1}, \quad y=h_{\alpha}(u) h_{\alpha}(v)^{-1}$. Assume $k(x)=k(y)$. Then $f(s) f(t)^{-1}=f(u) f(v)^{-1}$. Since $s, t, u, v$, are in $C_{\alpha}, f(s), f(t), f(u)$, $f(v), f(t)^{-1} f(v)^{-1}$ are in $f\left(C_{\alpha}\right)=T_{\alpha^{\prime}}$ a commutative semigroup. Thus $f(s) f(t)^{-1}=f(u) f(v)^{-1}$ implies $f(s) f(v)=f(u) f(t)$, or $f(s \circ v)=f(u \circ t)$. Since $f$ is $1-1, s \circ v=u \circ t$. Now $h(s \circ v)=h(u \circ t)$, or $h_{\alpha}(s) h_{\alpha}(v)=$ $h_{\alpha}(u) h_{\alpha}(t)$. Thus $x=y$.

Let $x=h_{\alpha}(s) h_{\alpha}(t)^{-1}, y=h_{\beta}(u) h_{\beta}(v)^{-1}$. Assume $k(x)=k(y)$. We prove that $\alpha=\beta$. Since $k$ restricted to $G_{\alpha}$ is $1-1$, this will prove $x=y$. Now $f(s) f(t)^{-1}=f(u) f(v)^{-1}$, where $s, t \in C_{\alpha}$ and $u, v \in C_{\beta}$. We proved $f\left(C_{\alpha}\right) \subseteq H \times\left\{\alpha^{\prime}\right\}$; similarly, $f\left(C_{\beta}\right) \subseteq H \times\left\{\beta^{\prime}\right\}$. Since $f(s) f(t)^{-1}=$ $f(u) f(v)^{-1}, \alpha^{\prime}=\beta^{\prime}$. If $\alpha \neq \beta$ then $f$ would be an injection of the noncommutative semigroup $C_{\alpha} \cup C_{\beta}$ into the commutative semigroup $H \times\left\{\alpha^{\prime}\right\}$. Thus $\alpha=\beta$.

CoROLlary 2.5. Let $S$ be an $R Z U$ of $F=\left\{C_{\alpha}: \alpha \in A\right\}$, where $F$ is a disjoint family of commutative cancellative semigroups. Then $S$ is left cancellative and left commutative.

Proof. By Theorem 2.4, $S$ can be thought of as a subsemigroup of a right abelian group. Every subsemigroup of a right abelian group is left cancellative and left commutative.

THEOREM 2.6. If a semigroup $S$ is left commutative and left cancellative, then $S$ has a quotient right abelian group.

Proof. Define a relation $\rho$ on $S$ by $x \rho y$ if and only if there exist $c, d \in S$ such that $c x=d y$. We prove that $\rho$ is an $r$-congruence on $S(S / \rho$ is a right zero semigroup), and each congruence class is commutative cancellative. Thus $S$ is an RZU of commutative cancellative semigroups and the result follows from the previous theorem. 
Now $\rho$ is certainly reflexive and symmetric.

Suppose $x \rho y$ and $y \rho z$. There exist $a, b, c, d$ in $S$ such that: $a x=$ $b y$ and $c y=d z$. Now $c a x=c b y$, and $b c y=b d z$. By left commutativity, $c b y=b c y$. Thus $c a x=b d z$, or $x \rho z$. Easily, $\rho$ is right compatible. Left compatibility follows from left commutativity.

Now xyoy, for let $c$ be arbitrary, and let $d=c x$; then $c x y=d y$. Thus $\rho$ is an $r$-congruence.

We now prove that each congruence class is commutative. Since $S$ is left cancellative, each congruence class will be commutative and cancellative.

Let $x \rho y$. We have $c x=d y$. Thus $c x d y=d y c x$. By left commutativity $c d x y=c d y x$. By left cancellativity $x y=y x$. Easily any congruence class of an $r$-congruence is a semigroup.

REMARK. Since each congruence class of $\rho$ is commutative, $\rho$ is the smallest $r$-congruence on $S$.

Every subsemigroup of a right abelian group is left commutative and left cancellative. Thus the last theorem characterizes subsemigroups of right abelian groups.

LEMma 2.7. Let $S$ be a left commutative semigroup. Define $\eta$ on $S$ by: $x \eta y$ if and only if there is an element $b$ in $S$ such that $b x=$ by. Then $\eta$ is the smallest left cancellative congruence on $S$.

Proof. Using left commutativity one proves $\eta$ is a congruence. It is also easy to prove that $S / \eta$ is left cancellative.

Let $f$ be a homomorphism of $S$ onto a left cancellative semigroup $S^{\prime}$. Suppose $x \eta y$, or $a x=a y$ for some $a$ in $S$; then $f(a x)=f(a y)$, or $f(a) f(x)=f(a) f(y)$. Since $S^{\prime}$ is left cancellative $f(x)=f(y)$. Let $\rho$ be the congruence induced by $f$. If $x \eta y$ then $x \rho y$, or $n \subseteq \rho$.

We now consider constructing an RZU of a family of homomorphic images given that the original family has an RZU.

Theorem 2.8. Let $S$ be an $R Z U$ of $\left\{C_{\alpha}: \alpha \in A\right\}$, where $C_{\alpha}$ are commutative semigroups. Let $\eta_{\alpha}$ be the smallest left cancellative congruence defined on $C_{\alpha}$. Then the family $\left\{C_{\alpha} \mid \eta_{\alpha}: \alpha \in A\right\}$ has an RZU.

Proof. Let $\eta_{\alpha}[x]$ be a congruence class of $C_{\alpha}$, and let $\eta_{\beta}[y]$ be a congruence class of $C_{\beta}$. Define $\eta_{\alpha}[x] \circ \eta_{\beta}[y]=\eta_{\beta}[x y] . \quad(x y$ is taken in S.) If the operation is well defined, then it is associative, and it defines an RZU of the $C_{\alpha} / \eta_{\alpha}$.

Suppose $\eta_{\alpha}[x]=\eta_{\alpha}[a]$, and $\eta_{\beta}[y]=\eta_{\beta}[b]$. We would like to show 
that $\eta_{\beta}[a b]=\eta_{\beta}[x y]$. Since $\eta_{\alpha}[x]=\eta_{\alpha}[a]$ there exists $d$ in $C_{\alpha}$ such that $d x=d a$. Similarly, there exists $w$ in $C_{\beta}$ such that $w y=w b$. Now $d x w y=d a w b$. All elements lie in the RZU of $C_{\alpha}$ and $C_{\beta}$. We invoke Lemma 2.1. By left commutativity, $d w x y=d w a b$. Thus $\eta_{\beta}[x y]=$ $\eta_{\beta}[a b]$, because $d w \in C_{\beta}$ as are $x y$ and $a b$.

Since $\left\{C_{\alpha} / \eta_{\alpha}: \alpha \in A\right\}$ has an RZU, by Theorem 2.4, the quotient groups of the $C_{\alpha} / \eta_{\alpha}$ are isomorphic. This imposes another necessary condition for a family of commutative semigroups to have an RZU. If $|A|=2$, using Lemma 2.1, then for $\eta$ of Lemma 2.7: $\eta=\eta_{1} \cup \eta_{2}$, $S / \eta=C_{1} / \eta_{1} \cup C_{2} / \eta_{2}$ RZU.

III. Necessary and sufficient conditions on commutative cancellative semigroups to have an RZU. This section begins by relating the translational semigroup of a commutative cancellative semigroup $A$ with the quotient group of $A$.

Definition 3.1. Let $A$ be a commutative semigroup. A function $f$, from $A$ into $A$, is called a translation of $A$ if $f(a b)=f(a) b$ for all $a$ and $b$ in $A$. $T(A)$ will denote the semigroup of all translations on $A$. Let $i$ be the mapping from $A$ into $T(A)$ given by $i(a)=$ $f_{a}$, where $f_{a}$ is the inner translation induced by $a \in A: f_{a}(x)=a x$, for all $x$ in $A$. $i(A)$ is the semigroup of all inner translations on $A$.

Let $A$ be a commutative cancellative semigroup. Let $G$ be the quotient group of $A$. Recall $G$ is abelian. $A$ may be injected into $G$ as a set of generators. Using this fact we relate $G$ to $T(A)$.

The following lemmas are easily proven.

Lemma 3.2. Let $A$ be injected by $j$ as a set of generators into $G$. Let $f \in T(A)$. Define $f^{*}$ on $j(A)$ by $f^{*}(j(a))=j(f(a))$. $f^{*}$ can be extended to a translation on $G$ as follows: if $g \in G$ there exists $j\left(a_{1}\right)$ and $j\left(a_{2}\right)$ such that $g=j\left(a_{1}\right) j\left(a_{2}\right)^{-1}$. Define $f^{*}(g)=f^{*}\left(j\left(a_{1}\right)\right) j\left(a_{2}\right)^{-1}$.

LemMA 3.3. Let $i: A \rightarrow T(A)$ given by: $i(a)=f_{a}$. Let $h: T(A) \rightarrow$

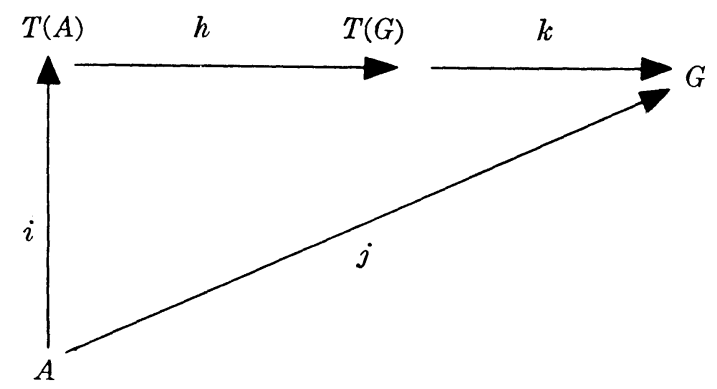

Figure 2 
$T(G)$ given by: $h(f)=f^{*}$. Let $k: T(G) \rightarrow G$ given by: $k\left(f^{*}\right)=f^{*}(1)$, where 1 is the identity of $G$. The above diagram commutes in the sense that $j(a)=k(h(i(a)))$ for all $a \in A$. Each map is injective; $k$ is onto.

Corollary 3.4. Let $A$ be a commutative cancellative semigroup. $T(A)$ is a commutative cancellative semigroup. If $f \in T(A)$ then $f$ is $1-1$ on $A$.

Proof. Since kh injects $T(A)$ into an abelian group, $T(A)$ is commutative and cancellative. Let $f \in T(A)$. Suppose that $f\left(a_{1}\right)=f\left(a_{2}\right)$. Then $j\left(f\left(a_{1}\right)\right)=j\left(f\left(a_{2}\right)\right)$, or $f^{*}\left(j\left(a_{1}\right)\right)=f^{*}\left(j\left(a_{2}\right)\right)$. $j$ is injective; also every translation on a group is $1-1$. Thus $j\left(a_{1}\right)=j\left(a_{2}\right)$, and $a_{1}=a_{2}$, or $f$ is $1-1$.

Lemma 3.5. Let $A$ be a commutative cancellative semigroup. Let $G$ be the quotient group of $A$. Let $j$ be an injection of $A$ into $G$ as a set of generators. Define $T G(A)=\{g \in G: g j(A) \leqq j(A)\}$. Under the injection kh of Lemma 3.3, $T(A) \cong T G(A)$. Also $i(A)$ is equal to $h^{-1} k^{-1}(j(A))$.

Proof. Let $g \in T G(A)$. Define $f$ on $A$ by $f(a)=j^{-1}(g j(a)), a \in A$. Then $f \in T(A)$, and $k h(f)=g$. Thus $T G(A) \subseteq k h(T(A))$. To prove the reverse inclusion, let $f \in T(A)$. Since $f^{*}$ is a translation, and $f^{*}(j(a))=j(f(a))$, we have $f^{*}(1) j(a)=f^{*}(1 \cdot j(a))=f^{*}(j(a))=j(f(a))$. Thus $f^{*}(1) j(A) \subseteq j(A)$, or $k h(f) \in T G(A)$. The remaining part of the lemma is proven by $k h(i(A))=j(A)$ (Lemma 3.3) and the fact that $k h$ is injective.

Theorem 3.6. Let $F=\left\{S_{\alpha}: \alpha \in \Gamma\right\}$ be a disjoint family of commutative cancellative semigroups. Let $\alpha \in \Gamma$, and let $P(\alpha)$ be the following statement: there exists $T_{a}=\left\{f_{\beta}: \beta \in \Gamma\right\}$, a family of injections (isomorphisms, into), where $f_{\beta}: S_{\beta} \rightarrow T\left(S_{\alpha}\right)$ for all $\beta$ in $\Gamma$, and where $f_{\gamma}\left(S_{\gamma}\right) f_{\beta}\left(S_{\beta}\right) \subseteq f_{\gamma}\left(S_{\gamma}\right) \cap f_{\beta}\left(S_{\beta}\right)$ for all $\gamma$ and $\beta$ in $\Gamma$. The following are equivalent:

(a) $F$ has an $R Z U$.

(b) For any $\alpha_{0} \in \Gamma, P\left(\alpha_{0}\right)$ holds.

(c) For some $\alpha_{0} \in \Gamma, P\left(\alpha_{0}\right)$ holds.

Furthermore, in (b) and (c) we may take $f_{\alpha_{0}}$ to be $i$, the natural map of $S_{\alpha_{0}}$ onto the inner translations of $S_{\alpha_{0}}$.

Proof. We first prove (a) implies (b). Let $S$ be an RZU of $F$, and let $\alpha_{0}$ be a fixed but arbitrary member of $\Gamma$. For each $x$ in $S$, let $f_{x}$ be the mapping of $S_{\alpha_{0}}$ into $S_{\alpha_{0}}$ given by $f_{x}(a)=x a$ for all $a$ in $S_{\alpha_{v}}$. The range of $f_{x}$ is contained in $S_{\alpha_{0}}$ because $S_{\alpha_{0}}$ is a left ideal of $S$. The following are true: 
(1) $f_{x} \in T\left(S_{\alpha_{0}}\right)$.

(2) Let $f$ be the mapping from $S$ into $T\left(S_{\alpha_{0}}\right)$ given by $f(x)=$ $f_{x}$. $f$ is a homomorphism and $f$ restricted to any $S_{\alpha}$ is $1-1$. Note that $f$ restricted to $S_{\alpha_{0}}$ is the map $i$.

(3) $f\left(S_{\alpha}\right)$ is an ideal of $f(S)$ for all $\alpha$ in $\Gamma$.

(1) is easily checked as is the first part of (2). Let $\alpha$ be an arbitrary member of $\Gamma$. We now prove that $f$ restricted to $S_{\alpha}$ is $1-1$. Let $a$ and $b$ be members of $S_{\alpha}$. Suppose $f(a)=f(b)$. Then $a x=b x$ for all $x \in S_{\alpha_{0}}$. But then $a x a=b x a$ for all $x \in S_{\alpha_{0}}$. Let $x_{0} \in$ $S_{\alpha_{0}}$. We have $a\left(x_{0} a\right)=b\left(x_{0} a\right)$. Now $a, b \in S_{\alpha}$, and $\left(x_{0} a\right) \in S_{\alpha}$ because $S_{\alpha}$ is a left ideal of $S$. Since $S_{\alpha}$ is cancellative $a=b$. We now prove (3) by Corollary 3.4, $T\left(S_{\alpha_{0}}\right)$ is commutaive. Thus $f(S)$ is commutative. Each $S_{\alpha}$ is a left ideal of $S$. Since $f$ is a homomorphism, $f\left(S_{\alpha}\right)$ is a left ideal of $f(S)$. But all left ideals of a commutative semigroup are ideals.

For each $\alpha$ in $\Gamma$, let $f_{\alpha}$ be the restriction of $f$ to $S_{\alpha}$. Then $f_{\alpha}$ : $S_{\alpha} \rightarrow T\left(S_{\alpha_{0}}\right)$. $f_{\alpha}$ is an injection by (2). By (3) $f_{\alpha}\left(S_{\alpha}\right)$ and $f_{\beta}\left(S_{\beta}\right)$ are ideals of $f(S)$. Thus $f_{\alpha}\left(S_{\alpha}\right) f_{\beta}\left(S_{\beta}\right) \subseteq f_{\alpha}\left(S_{\alpha}\right) \cap f_{\beta}\left(S_{\beta}\right)$. This completes the proof of (a) implies (b).

Trivially (b) implies (c). We now prove (c) implies (a). Let $p\left(\alpha_{0}\right)$ hold. Define a binary operation on $F$ as follows: Let $x \in S_{\alpha}$ and $y \in S_{\beta}$.

$$
\begin{aligned}
& x \circ y=f_{\beta}^{-1}\left(f_{\alpha}(x) f_{\beta}(y)\right) \\
& y \circ x=f_{\alpha}^{-1}\left(f_{\beta}(y) f_{\alpha}(x)\right)
\end{aligned}
$$

where $f_{\alpha}, f_{\beta} \in T_{\alpha_{0}}$. The operation is well defined because $f_{\alpha}(x) f_{\beta}(y) \in$ $f_{\alpha}\left(S_{\alpha}\right) f_{\beta}\left(S_{\beta}\right) \subseteq f_{\alpha}\left(S_{\alpha}\right) \cap f_{\beta}\left(S_{\beta}\right)$. Thus $f_{\alpha}(x) f_{\beta}(y) \in f_{\beta}\left(S_{\beta}\right)$, and we may apply $f_{\beta}^{-1}$. Similarly $f_{\beta}(y) f_{\alpha}(x) \in f_{\alpha}\left(S_{\alpha}\right)$. The operation restricted to any $S_{\alpha}$ is the semigroup operation already given on $S_{\alpha}$. Let $x, y \in S_{\alpha}$. Then $x \circ y=f_{\alpha}^{-1}\left(f_{\alpha}(x) f_{\alpha}(y)\right)=f_{\alpha}^{-1}\left(f_{\alpha}(x y)\right)=x y$. This is true because $f_{\alpha}$ is an injection. If the operation is associative, it certainly defines an RZU of $F$.

Let $x \in S_{\alpha}, y \in S_{\beta}$, and $z \in S_{\gamma}$. Then $(x \circ y) \circ z=\left(f_{\beta}^{-1}\left(f_{\alpha}(x) f_{\beta}(y)\right)\right) \circ z=$ $f_{\gamma}^{-1}\left(f_{\beta}\left(f_{\beta}^{-1}\left(f_{\alpha}(x) f_{\beta}(y)\right)\right) f_{\gamma}(z)\right)=f_{\gamma}^{-1}\left(\left(f_{\alpha}(x) f_{\beta}(y)\right) f_{\gamma}(z)\right)$. Similarly $\quad x \circ(y \circ z)=$ $f_{\gamma}^{-1}\left(f_{\alpha}(x)\left(f_{\beta}(y) f_{\gamma}(z)\right)\right)$. Now $(x \circ y) \circ z=x \circ(y \circ z)$ since $f_{\alpha}(x)\left(f_{\beta}(y) f_{\gamma}(z)\right)=$ $\left(f_{\alpha}(x) f_{\beta}(y)\right) f_{\gamma}(z)$. The above product is taken in the semigroup $T\left(S_{\alpha_{0}}\right)$, and is in $f_{\gamma}\left(S_{\gamma}\right)$.

REMARK. Let $(\alpha, \beta) \in \Gamma \times \Gamma$. Because $f_{\alpha}\left(S_{\alpha}\right)$ and $f_{\beta}\left(S_{\beta}\right)$ are subsets of the commutative semigroup $T\left(S_{\alpha_{0}}\right), f_{\alpha}\left(S_{\alpha}\right) f_{\beta}\left(S_{\beta}\right) \subseteq f_{\alpha}\left(S_{\alpha}\right) \cap f_{\beta}\left(S_{\beta}\right)$ implies the same condition for the pair $(\beta, \alpha)$. Thus we need only consider one condition.

We restate Theorem 3.6 for two semigroups as follows: $F=\{A, B\}$ has an RZU if and only if there exists an injection $f$ from $B$ into 
$T(A)$ such that $f(B) i(A) \cong f(B) \cap i(A)$.

CoRollary 3.7. Let $F=\left\{S_{\alpha}: \alpha \in A\right\}$ be a disjoint family of commutative cancellative semigroups. If for some $\alpha_{0} \in A$ each $S_{\alpha}$ is isomorphic to an ideal of $S_{\alpha_{0}}$ then $F$ has an $R Z U$.

Proof. To say $S_{\alpha}$ is isomorphic to an ideal of $S_{\alpha_{0}}$ means there exists $h_{\alpha}: S_{\alpha} \rightarrow S_{\alpha_{0}}$, where $h_{\alpha}$ is an injection, and $h_{\alpha}\left(S_{\alpha}\right)$ is an ideal of $S_{\alpha_{0}}$. Let $f_{\alpha}: S_{\alpha} \rightarrow T\left(S_{\alpha_{0}}\right)$, given by $f_{\alpha}=i_{\alpha_{0}} \circ h_{\alpha}$, where $i_{\alpha_{0}}: S_{\alpha_{0}} \rightarrow T\left(S_{\alpha_{0}}\right)$, given by $i_{\alpha_{0}}(x)=f_{x}$. $\left\{f_{\alpha}: \alpha \in A\right\}$ satisfies (c) of Theorem 3.6 because $f_{\alpha}\left(S_{\alpha}\right)$ is an ideal of $i_{\alpha_{0}}\left(S_{\alpha_{0}}\right)$.

Corollary 3.8. Let $A$ and $B$ be two disjoint commutative cancellative semigroups having an $R Z U$. If $A$ is a group then $B$ is a group, and $A \cong B$.

Proof. Every translation of a group is inner; thus $T(A)=i(A)$. Now $i(A)$ is the regular representation of $A$; thus $i(A) \cong A$. By Theorem 3.6, there exists an injection $f$ of $B$ into $T(A)$ such that $f(B) i(A) \subseteq f(B) \cap i(A) . \quad f$ is an injection into $i(A)$. Since $T(A)$ is commutative, $f(B)$ is an ideal of $i(A)$. But a group has no proper ideals. Thus $f(B) \cong i(A)=A$. Since $f$ is an injection $B \cong A$.

We now give an interpretation of Theorem 3.6 in terms of quotient groups. Let $A$ be a commutative cancellative semigroup. Let $j$ be an injection of $A$ as a set of generators into $G$, the quotient group of $A$. Let $f$ be the isomorphism from $T(A)$ onto $T G(A)(T G(A)$ of Lemma 3.5; $f=k$ h of Lemma 3.3). Let $B$ be a commutative cancellative semigroup having an RZU with $A$. Let $h$ be an injection of $B$ into $T(A)$ such that $h(B) i(A) \subseteq h(B) \cap i(A)$. Compose the maps $h$ and $f$. We have $(f h)(B) j(A) \subseteq(f h)(B) \cap j(A)$. Evidently, $B$ is isomorphic to $B^{\prime}$, a subsemigroup of $T G(A)$ such that $B^{\prime} j(A) \cong B^{\prime} \cap j(A)$. Conversely, an isomorphic copy of such a $B^{\prime}$ will have an RZU with $A$. Thus we have a way of finding all commutative cancellative semigroups having an RZU with $A$.

\section{REFERENCES}

1. A. H. Clifford, and G. B. Preston, The Algebraic Theory of Semigroups, Vol. 1, Math. Surveys, No. 7, Amer. Math. Soc., Providence, R. I. (1961).

2. M. Petrich, The Structure of a Class of Semigroups Which Are Unions of Groups, Notices Amer. Math. Soc., 12, No. 1, Part 1 (1965), 102.

3. R. Yoshida, l-Compositions of Semigroups I, Memoirs of the Research Inst. of Science and Eng., Ritsumeikan University, 14 (1965) 1-12. 
4. R. Yoshida, l-Compositions of Semigroups II, Memoirs of the Research Inst. of Science and Eng., Ritsumeikan University, 15, (1966), 1-5.

Received January 7, 1971 and in revised form June 28, 1971. The contents of this paper were taken from the author's $\mathrm{Ph}$. D. thesis.

University of CALIFornia, Davis

AND

University of California, LawRence Radiation Lab., Livermore 


\section{PACIFIC JOURNAL OF MATHEMATICS}

\section{EDITORS}

\author{
H. SAMELSON \\ Stanford University \\ Stanford, California 94305

\section{R. HoвBY} \\ University of Washington \\ Seattle, Washington 98105
}

\section{J. DugundJI}

Department of Mathematics University of Southern California Los Angeles, California 90007

\author{
RichaRd ARENS \\ University of California \\ Los Angeles, California 90024
}

\section{ASSOCIATE EDITORS}
E. F. BECKENBACH
B. H. NEUMANN
F. WOLF
K. YoshidA

\section{SUPPORTING INSTITUTIONS}

\author{
UNIVERSITY OF BRITISH COLUMBIA \\ CALIFORNIA INSTITUTE OF TECHNOLOGY \\ UNIVERSITY OF CALIFORNIA \\ MONTANA STATE UNIVERSITY \\ UNIVERSITY OF NEVADA \\ NEW MEXICO STATE UNIVERSITY \\ OREGON STATE UNIVERSITY \\ UNIVERSITY OF OREGON \\ OSAKA UNIVERSITY
}

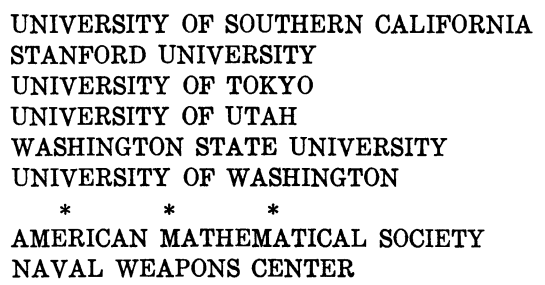

The Supporting Institutions listed above contribute to the cost of publication of this Journal, but they are not owners or publishers and have no responsibility for its content or policies.

Mathematical papers intended for publication in the Pacific Journal of Mathematics should be in typed form or offset-reproduced, (not dittoed), double spaced with large margins. Underline Greek letters in red, German in green, and script in blue. The first paragraph or two must be capable of being used separately as a synopsis of the entire paper. The editorial "we" must not be used in the synopsis, and items of the bibliography should not be cited there unless absolutely necessary, in which case they must be identified by author and Journal, rather than by item number. Manuscripts, in dup icate if possible, may be sent to any one of the four editors. Please classify according to the scheme of Math. Rev. Index to Vol. 39. All other communications to the editors should be addressed to the managing editor, Richard Arens, University of California, Los Angeles, California, 90024.

50 reprints are provided free for each article; additional copies may be obtained at cost in multiples of 50 .

The Pacific Journal of Mathematics is published monthly. Effective with Volume 16 the price per volume (3 numbers) is $\$ 8.00$; single issues, $\$ 3.00$. Special price for current issues to individual faculty members of supporting institutions and to individual members of the American Mathematical Society: $\$ 4.00$ per volume; single issues $\$ 1.50$. Back numbers are available.

Subscriptions, orders for back numbers, and changes of address should be sent to Pacific Journal of Mathematics, 103 Highland Boulevard, Berkeley, California, 94708.

PUBLISHED BY PACIFIC JOURNAL OF MATHEMATICS, A NON-PROFIT CORPORATION

Printed at Kokusai Bunken Insatsusha (International Academic Printing Co., Ltd.), 270, 3-chome Totsuka-cho, Shinjuku-ku, Tokyo 160, Japan. 


\section{Pacific Journal of Mathematics}

\section{Vol. 41, No. 2 December, 1972}

Tom M. (Mike) Apostol, Arithmetical properties of generalized Ramanujan sums .......................................... 281

David Lee Armacost and William Louis Armacost, On p-thetic groups ........ 295

Janet E. Mills, Regular semigroups which are extensions of groups .......... 303

Gregory Frank Bachelis, Homomorphisms of Banach algebras with minimal ideals ................................................ 307

John Allen Beachy, A generalization of injectivity .................. 313

David Geoffrey Cantor, On arithmetic properties of the Taylor series of rational functions. II.........................................

Václáv Chvátal and Frank Harary, Generalized Ramsey theory for graphs. III.

Small off-diagonal numbers .................................. 335

Frank Rimi DeMeyer, Irreducible characters and solvability of finite groups . . . . 347

Robert P. Dickinson, On right zero unions of commutative semigroups........ 355

John Dustin Donald, Non-openness and non-equidimensionality in algebraic

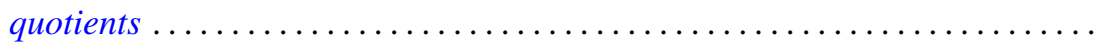

John D. Donaldson and Qazi Ibadur Rahman, Inequalities for polynomials with a prescribed zero ........................................ 375

Robert E. Hall, The translational hull of an $N$-semigroup ................ 379

John P. Holmes, Differentiable power-associative groupoids.............. 391

Steven Kenyon Ingram, Continuous dependence on parameters and boundary data for nonlinear two-point boundary value problems .

Robert Clarke James, Super-reflexive spaces with bases ..........

Gary Douglas Jones, The embedding of homeomorphisms of the plane in

continuous flows...............................

Mary Joel Jordan, Period $H$-semigroups and $t$-semisimple periodic

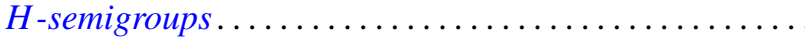

Ronald Allen Knight, Dynamical systems of characteristic 0

Kwangil Koh, On a representation of a strongly harmonic ring by sheaves...

Hui-Hsiung Kuo, Stochastic integrals in abstract Wiener space. ..

Thomas Graham McLaughlin, Supersimple sets and the problem of extending a

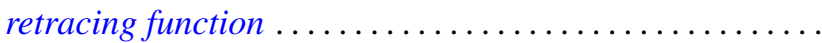

William Nathan, Open mappings on 2-manifolds .

M. J. O'Malley, Isomorphic power series rings

Sean B. O'Reilly, Completely adequate neighborhood systems and metrization

Qazi Ibadur Rahman, On the zeros of a polynomial and its derivative...

Russell Daniel Rupp, Jr., The Weierstrass excess function ..

Hugo Teufel, A note on second order differential inequalities and functional

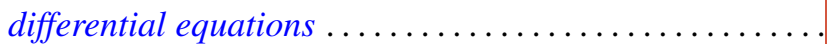

M. J. Wicks, A general solution of binary homogeneous equations over free 